\title{
Факторы интенсификации кипения в двухфазных системах терморегулирования
}

\author{
Б. B. Kocoù
}

Одесская национальная академия пищевых технологий, ул. Канатная, 112, Одесса, 65039, Украина

$\triangle$ e-mail: bkosoy@gmail.com

Работа посвящена экспериментальному и теоретическому решению важной научно-технической задачи интенсификации теплообмена в микроструктурных элементах систем терморегулирования с иелью повышения их теплотехнической эффективности, надежности, уменьшения массы и габаритов. Рассмотрены основные структурные характеристики капиллярно-пористых тел, такие как эффективная пористость, кривая распределения пор по радиусу, проницаемость и т.д. Определены основные механизмы переноса массы вещества в пористой среде. Выполнен анализ особенностей модельных представлений для процессов кипения жидкости на пористых и на развитых поверхностях теплообмена и обоснованы физические факторы, позволяющие обеспечить высокие тепловые потоки при мальх разностях температур. Реализованный комплекс экспериментальных и расчётных исследований характеристик процессов двухфазного теплообмена при кипении в микроканальных тонкопленочных испарителях позволил определить специфику влияния структуры поверхности на интенсивность процесса теплоотдачи, установлены зависимости их теплопередающей способности от формь поперечных сечений и соотночений между глубиной и шириной прямоугольных микроканалов. Проведён теоретический анализ особенностей гидродинамических и теплообменных процессов, протекающих в микроструктурах, продемонстрировавиий возможности интенсификации теплообмена при кипении путём оптимизации теплотехнических характеристик микроструктуры и использования гибридных микроструктур различной пористости. Установлено, что процесс теплообмена при испарении и кипении жидкостей в капиллярнопористых телах и на развитых поверхностях, покрытых сетью капиллярных каналов, обладает рядом особенностей по сравнению с кипением жидкости в большом объеме над гладкой поверхностью, а также в каналах и трубах с гладкими стенками. Пористая структура и капиллярные канавки интенсифицируют процесс теплообмена в широком диапазоне тепловых потоков и позволяют производить плавный переход от режима испарения к режиму кипения. Процесс наступления кризиса кипения сглаживается, при этом кривая кипения не имеет ярко выраженных максимумов. Покрытие пористыми структурами или микроканалами поверхности теплообмена с изелью интенсификации процесса особенно эффективно для криогенных жидкостей (гелий, водород и т. д.), а также в низкотемпературных тепловых трубах и термосифонах.

Ключевые слова: Интенсификация; Кипение; Терморегулирование; Микроканаль; Пористые среды

\section{Фактори інтенсифікації кипіння в двофазних системах терморегулювання}

\section{Б. В. Косой}

Одеська національна академія харчових технологій, вул. Канатна, 112, Одеса, 65039, Україна

Робота присвячена експериментальному і теоретичному вирішенню важливої науково-технічної задачі інтенсифікації теплообміну в мікроструктурованих елементах систем терморегулювання з метою підвищення їх теплотехнічної ефективності, надійності, зменшення маси і габаритів. Розглянуто основні структурні характеристики капілярно-пористих тіл, такі як ефективна пористість, крива розподілу пір за радіусом, проникність і т.д. Визначено основні механізми перенесення маси речовини в пористому середовищі. Виконано аналіз особливостей модельних уявлень для процесів кипіння рідини на пористих $і$ на розвинених поверхнях теплообміну і обтрунтовані фізичні фактори, щьо дозволяють забезпечити високі теплові потоки при малих різницях температур. Реалізований комплекс експериментальних і розрахункових досліджень характеристик проиесів двухфазного теплообміну при кипінні в мікроканальних тонкоплівкових випарниках дозволив визначити специифіку впливу структури поверхні на інтенсивність процесу тепловіддачі, встановлені залежності їх теплопередаючої здатності від форми поперечних перерізів і співвідношень між глибиною $і$ шириною прямокутних мікроканалів. Проведено теоретичний аналіз особливостей гідродинамічних і теплообмінних прочесів, щчо протікають в мікроструктурах, який продемонстрував можливості інтенсифікації теплообміну при кипінні шляхом оптимізації теплотехнічних характеристик мікроструктури і використання гібридних мікроструктур різної пористості. Встановлено, щуо процес теплообміну при випаровуванні і кипінні рідин в капілярно-пористих тілах і на розвинених поверхнях, покритих мережею капілярних каналів, має низку особливостей порівняно з кипінням рідини в великому об'ємі над гладкою поверхнею, а також в каналах і трубах з гладкими стінками. Пориста структура і капілярні канавки інтенсифікують процес теплообміну в широкому діапазоні теплових потоків і дозволяють здійснити плавний перехід від режиму випаровування до режиму кипіння. Процес настання 
кризи кипіння згладжується, при изьому крива кипіння не має яскраво виражених максимумів. Покриття пористими структурами або мікроканалами поверхні теплообміну з метою інтенсифікації процесу є особливо ефективним для кріогенних рідин (гелій, водень $і$ т. п.), а також в низькотемпературних теплових трубах $і$ термосифонах.

Ключові слова: інтенсифікацุія, кипіння, терморегулювання, мікроканали, пористі середовища

(C) The Author(s) 2018. This article is an open access publication

This work is licensed under the Creative Commons Attribution 4.0 International License (CC BY) http://creativecommons.org/licenses/by/4.0/

\section{1. Введение}

В настоящее время все большее распространение приобретают методы охлаждения энергонапряженных малогабаритных источников тепла (генераторные лампы, микропроцессоры, лазеры и т. д.), основанные на использовании процесса кипения на развитых (оребренных) поверхностях нагрева, когда плотность тепловых потоков на поверхности, несущей ребра или канавки, в несколько раз превышает величину, характерную для гладкой поверхности. Однако в литературе не имеется достаточного количества данных о закономерности процесса кипения на затопленных развитых поверхностях нагрева, омываемых потоком насыщенной или недогретой жидкости.

Микроструктура различных материалов имеет большое значение для определения оптимальных режимов ряда технологических процессов, таких, как двухфазное терморегулирование, охлаждение фильтрацией однофазной жидкости, осуществление химических реакций в пористых катализаторах. Микроструктурные элементы, типичными представителями которых являются микротепловые трубки, капиллярнопористые фитили, микроканальные теплообменникииспарители, микронасосы и т.п. находят все более широкие приложения в системах обеспечения тепловых режимов радиоэлектроники, приборостроения, химической промышленности, космических аппаратов и медицинской техники. При этом, если на начальном этапе особое внимание уделялось разработке эффективных способов изготовления таких микроустройств, то в настоящее время большее число исследований направлено на разработку перспективных методов надёжного прогнозирования их функциональности, транспортных и теплотехнических характеристик, структурной целостности и оптимальности процессов.

\section{2. Основные структурные характеристики ка- пиллярно-пористых тел}

Под пористой средой обычно подразумевают твердое тело, содержащее поры в виде пустых промежутков сложной формы, равномерно расположенных по объему тела Поры могут быть сообщающимися или замкнутыми. Доля сообщающихся друг с другом пор составляет эффективную пористость системы. Часто эффективная пористость твердого тела намного меньше его общей пористости. Рассмотрим основные структурные характеристики капиллярно-пористых тел.
Пористость $\Pi_{v}$ определяется как отношение объема пор $V_{\text {nор }}$ к объему тела $V_{\text {тел}}$. Существует также понятие поверхностной пористости или просвета $\Pi_{s}$, представляющей собой отношение площади пор $\mathrm{A}_{\text {nор }}$ к площади поверхности тела $\mathrm{A}_{\text {тел }}$ :

$$
\Pi_{v}=V_{\text {nop }} / V_{\text {meл }} ; \Pi_{s}=\mathrm{A}_{\text {nop }} / \mathrm{A}_{\text {meл }} .
$$

Эффективная пористость определяется как отношение объема сообщающихся пор к общему объему тела:

$$
\Pi_{э ф}=V_{\text {эф.nор }} / V_{\text {тел }} .
$$

Простейшими моделями пористого тела являются система, содержащая сферы одинакового размера, плотно прижатые друг к другу, а также пучок параллельных капилляров.

Под удельной поверхностью пористого тела понимают площадь внутренних поверхностей пор, приходящуюся на единицу объема материала. Удельная поверхность определяет проницаемость капиллярнопористого тела, т. е. способность пропускать через себя жидкость: Кроме твердых пористых тел, в природе существуют дисперсные и волокнистые тела. Наличие в теле пор различных геометрических размеров учитывается функцией распределения пор по размерам, а для дисперсных систем - кривой распределения частиц по размерам. Иногда для характеристики дисперсных (зернистых) систем пользуются средним размером (диаметром) частиц и дисперсией.

Наряду с пористостью и кривой распределения пор по радиусу существенное значение имеет такая характеристика пористого тела, как проницаемость $K$. Проницаемость - это свойство пористого материала пропускать через себя жидкость под действием градиента внешнего давления, т. е. проводимость тела по отношению к жидкости. Проницаемость $K$ связана с эффективной пористостью тела и размерами пор и обычно определяется из закона Дарси. Проницаемость является макроскопической характеристикой пористого тела:

$$
K=\frac{m}{\mathrm{~A}(\Delta \mathrm{P} / l)}
$$

где А - площадь поперечного сечения; $m$ - вязкость жидкости; 1 - толщина тела; $\Delta \mathrm{P}$ - перепад давления на образце. 
При больших скоростях фильтрации жидкости (турбулентное течение в порах) имеет место отклонение от закона Дарси (двухчленный закон фильтрации)

$$
\Delta \mathrm{P}=-\frac{\eta}{\mathrm{K}} \vec{v}-\frac{\beta \rho v}{\sqrt{\mathrm{K}}} \vec{v},
$$

в котором коэффициент сопротивления зависит от скорости движения жидкости (второй член уравнения).

Пористые тела, содержащие в себе жидкость или газ, под действием нагрева или охлаждения могут изменять свои физические свойства.

Вид связи жидкости с капиллярно-пористым телом важная характеристика. Процесс удаления жидкости из пор сопровождается нарушением ее связи со стенками. По энергии связи можно сделать следующие разграничения: химическая связь, физико-химическая и физикомеханическая. Для криогенных жидкостей наиболее распространены две последние связи. К физикохимической относят адсорбционную связь вещества со стенками пор и осмотическое поглощение жидкости, к физико-механической - связь жидкости со стенками капилляров и жидкость смачивания. Жидкость, находящаяся в капиллярах и ограниченная менисками кривой поверхности раздела жидкость - газ (пар), является свободной жидкостью, за исключением тончайшего слоя, адсорбционно связанного со стенкой твердого тела. Понижение давления пара над поверхностью мениска формально характеризует энергию связи капиллярной жидкости $\mathrm{A}(r)$, определяемой действием искривленного мениска, и не зависит от свойств твердого тела в условиях полного смачивания и количества жидкости в капиллярах:

$$
\mathrm{A}(r)=\frac{2 \sigma}{r^{\prime}} V_{0}
$$

где $V_{0}$ - удельный объем; $\sigma$ - коэффициент поверхностного натяжения; $r^{\prime}$ - радиус искривленного мениска.

Капиллярное давление жидкости в элементарных капиллярах находят по формуле Лапласа

$$
\mathrm{P}_{h}=\sigma\left(\frac{1}{r_{1}^{\prime}}+\frac{1}{r_{2}{ }^{\prime}}\right)=2 \sigma\left(\frac{1}{r^{\prime}}\right)
$$

где $r_{1}{ }^{\prime}$ и $r_{2}{ }^{\prime}$ - главные радиусы кривизны мениска;

$$
\text { ( } \left.1 / r^{\prime}\right) \text { - средняя кривизна мениска. }
$$

В стационарном состоянии капиллярное давление уравновешивается гидростатическим столбом жидкости в капилляре:

$$
h=\frac{\mathrm{P}_{h}}{g \Delta \rho}=\frac{2 \sigma}{g \Delta \rho}\left(\frac{1}{r^{\prime}}\right)
$$

Между радиусом элементарного цилиндрического капилляра и средним радиусом мениска $r^{\prime}$ имеется зависимость $r=r^{\prime} \cos \theta$. Для криогенных жидкостей $\theta$ близок к нулю, поэтому $r=r^{\prime}$. Капиллярный потенциал обычно записывают в виде

$$
\varphi_{h}=h_{g}=\frac{\mathrm{P}_{h}}{\Delta \rho}=\frac{2 \sigma}{\Delta \rho}\left(\frac{1}{r^{\prime}}\right)
$$

Для криогенных жидкостей $\varphi_{h}<0$, так как $1 / r^{\prime}$ для вогнутого мениска отрицателен.

По А.В. Лыкову [1], наиболее важной структурной характеристикой пористой среды является дифференциальная кривая поверхностного распределения проницаемости $f_{s}(r)$, аналогичная дифференциальной кривой распределения пор по поверхности $M$ ). Однородная среда характеризуется такой функцией, где $\mathrm{A}_{\mathrm{i}}-$ постоянная, удовлетворяющая условию $\sum_{l=1}^{N} \mathrm{~A}_{i}=1 ; \mathrm{N}-$ конечная величина. Если $f(K)$ не может быть представлена конечным числом функций $\mathrm{A}_{i}$, то среда называется неоднородной. Если $f(K)$ мономодельна, то среда будет гомогенной, если же среда описывается двумя или большим количеством кривых распределения $f(K)$, то среда гетерогенна.

Механизмы переноса массы вещества в пористой среде могут быть разными:

- молекулярная диффузия при больших масштабах времени;

- турбулентный перенос в виде миграции вихрей;

- перемешивание, вызванное препятствиями;

- наличие автокорреляции в потоке в результате несвязности среды;

- рециркуляции, вызванные участками пониженного давления;

- адсорбция (стенками);

- вязкостное диффузионное и тепловое скольжение (дрейф вблизи стенок);

- макроскопическая дисперсия, вызванная неидеальностями, изменяющая общие линии тока.

Все виды массопереноса можно условно назвать дисперсией, что означает хаотическое рассеивание массы в пористой среде.

В изотермических условиях перенос массы описывается дифференциальным уравнением диффузии с наличием конвективного члена. Для одномерной задачи это уравнение имеет вид

$$
\frac{\partial \omega}{\partial \tau}=D^{\prime} \frac{\partial^{2} \omega}{\partial X^{2}}-v_{x} \frac{\partial \omega}{\partial x}
$$

где $D^{\prime}$ - коэффициент дисперсии; $v_{\mathrm{x}}$ - скорость конвективного переноса, определяемого законом Дарси. Коэффициент $D^{\prime}$ определяется по формуле ТейлораАриса:

$$
D^{\prime}=D_{\mu}+\frac{d_{\ni}^{2} v_{\phi}^{2}}{192 D_{\mu}} ;
$$

где $D_{м}-$ коэффициент молекулярной диффузии; $v_{\phi}-$ средняя скорость фильтрации: $d_{3}$ - диаметр эквивалентной трубки. 
Наиболее детально исследован процесс теплообмена при кипении жидкости на пористых и развитых поверхностях теплообмена. Мало работ посвящено процессу теплообмена при кипении жидкости внутри пористых тел, в частности при объемном тепловыделении (электрический нагрев тела, радиоактивное излучение и т. д.)

\section{3. Теплообмен при кипении жидкости на пори- стой поверхности}

В 1970 г. В.В. Ягов и Д.А. Лабунцов [2] опубликовали работу, посвященную анализу условий зарождения паровой фазы на шероховатой либо пористой поверхности теплообмена. Возникновение паровой фазы зависит от изменения термодинамического потенциала системы

$$
\Delta \Phi=\left(f_{n}-f_{m}\right) V \rho_{n}+\sigma \mathrm{A}\left[1-\frac{\mathrm{A}_{0}}{\mathrm{~A}}(1-\cos \theta)\right]
$$

Чем меньше приращение термодинамического потенциала $\Delta \Phi$, тем легче образуется паровой пузырь. Стабилизация процесса кипения и интенсификация процесса теплообмена могут быть достигнуты за счет уменьшения поверхности нагрева А и увеличения отношения $\mathrm{A}_{0}$ / А. Это увеличение достигается переходом от гладкой поверхности к пористой или развитой (канавки, щели и т. д.) а также путем специальной обработки поверхности (гидрофобизация, травление и т. д.). Изменение угла смачивания существенно сказывается на величине изменения $\Delta \Phi$. Обработка поверхности теплообмена, в частности гидрофобизация, может изменить угол смачивания и интенсифицировать теплообмен. Пористые структуры существенно стабилизируют процесс кипения жидкости, особенно при пониженных давлениях окружающей среды. Интенсификация процесса теплообмена при кипении криогенных жидкостей (азот, кислород и т. д.) на металлической поверхности теплообмена достигается благодаря слою инея, т. е. пористому покрытию из льда более высококипящей жидкости (вода, спирт и т.д.). Замечено, что металлические тела, мгновенно погружаемые в криогенную жидкость (азот, кислород), охлаждаются более быстро, если они покрыты тонким слоем пористого полимера или смазки.

Систематические исследования процесса кипения жидкости в пористых средах начали проводиться с появлением тепловых труб, испарители которых были выполнены из металлокерамики, войлока, сеток или в виде канавок. Испытывались пористые металлические и неметаллические структуры (пластины и трубы), вертикально или горизонтально расположенные и погруженные в жидкость частично либо полностью. Жидкость к поверхности теплообмена подавалась с помощью капиллярных сил или путем свободной конвекции. Объектом исследования служили вода, органические жидкости, жидкие металлы.

Пористые структуры являются системами с регулярными центрами ядрообразования, начало пузырькового кипения в них наступает при значительно меньших тепловых потоках по сравнению с гладкой поверхностью. Поскольку эффективная теплопроводность большинства металлических пористых структур, насыщенных неметаллическими жидкостями, превышает теплопроводность слоя жидкости эквивалентной толщины, перепады температуры па пористой структуре меньше.

При кипении на гладкой поверхности имеет место турбулентное перемешивание, в то время как в пористой структуре поле капиллярных сил организует процесс ламинарного течения жидкости по порам и капиллярам. При небольших величинах тепловых потоков процесс переноса энергии через пористую структуру осуществляется теплопроводностью и конвекцией жидкости в порах. При более высоких тепловых потоках начинается ядрообразование.

При наличии специальных паропроводящих каналов в пористом теле (перфорация, канавки и т. д.) пузырьки пара сравнительно легко выходят из пористой структуры, что позволяет избежать кризиса кипения при достаточно больших тепловых потоках (на воде до $1-1300$ кВт/м². При отсутствии пароотводящих каналов пузырьки пара скапливаются в зоне между поверхностью нагрева и пористой структурой и образуют пленку пара, толщина которой близка размеру пор или частиц, из которых спечена пористая структура. При дальнейшем увеличении теплового потока передача тепла осуществляется теплопроводностью через пленку пара к насыщенной жидкости в порах. Таким образом, при кипении жидкости в пористом теле не наблюдается зависимости теплового потока от разности температур $\left(\mathrm{T}_{\mathrm{c}}-\mathrm{T}_{\text {нас }}\right)$ в виде кривой Нукиямы (с двумя максимумами), характерной для кипения жидкости в большом объеме над гладкой поверхностью. Зависимость $\mathrm{A}_{i}$ от $\left(\mathrm{T}_{\mathrm{c}}-\mathrm{T}_{\text {нас }}\right)$ при кипении жидкости в пористой структуре имеет вид выпуклой кривой со слабо выраженным максимумом, величина которого зависит от свойств пористой структуры, часто $\mathrm{A}_{i}$ является постоянной величиной в широком диапазоне изменения $\left(\mathrm{T}_{\mathrm{c}}-\mathrm{T}_{\text {нас }}\right)$. Данная модель процесса кипения характерна для неметаллических жидкостей, имеющих теплопроводность, на порядок меньшую теплопроводности жидких металлов. Известно, что для начала вскипания необходим перегрев жидкости. При заданном перегреве жидкости до температуры Т в ней возможно существование равновесных пузырьков радиуса $r_{\kappa p}$. Пузырьки меньшего радиуса будут уменьшаться, а большего - расти. Критический размер пузыря почти линейно уменьшается с увеличением давления жидкости. Для металлов критический размер пузыря значительно больше, чем для неметаллических жидкостей. В связи с этим, процесс кипения в пористом теле затруднен по сравнению с неметаллическими жидкостями. Процесс кипения жидкого металла в пористых структурах имеет ряд особенностей и в данной статье не рассматривается.

Коэффициент теплообмена при кипении жидкости в пористом теле является консервативной величиной, мало зависящей от температуры и величины теплового потока (ниже критической). Процесс теплообмена отличается стабильностью, при одинаковых тепловых потоках перепады температур $\left(\mathrm{T}_{\mathrm{c}}-\mathrm{T}_{\text {нас }}\right)$ в пористых телах намного меньше, чем при кипении жидкости в большом объеме. Толщина паровой пленки приблизительно равна характерному размеру пор. Основным 
лимитирующим фактором теплообмена при кипении являются ограниченные возможности поля капиллярных сил по подаче жидкости к зоне нагрева:

$$
q_{\text {крит. }}=\frac{j r^{*}}{\mathrm{~A}_{u}},
$$

где $\mathrm{A}_{u}$ - площадь испарителя.

Для плоской поверхности теплообмена и гомогенного пористого фитиля авторы работы [3] предложили формулу для определения критического теплового потока

$$
q_{\text {крит }}=\frac{g\left[h_{\max }\left(\mathrm{T}_{i}\right) \frac{\sigma_{\varkappa}}{\sigma_{\varkappa}\left(\mathrm{T}_{i}\right)} \rho_{\varkappa}\left(\mathrm{T}_{i}\right)-\rho_{\varkappa} L \sin \gamma\right]}{\frac{L_{u} \mu_{\varkappa}}{\rho_{\varkappa} K^{\prime} r^{*} \xi b}\left(\frac{L_{u}}{2}+L_{a}\right)} ;
$$

где $L_{u}, L_{a}$-длина испарителя и адиабатической зоны, соответственно; $h_{\text {max }}$ - максимальная высота поднятия жидкости при заданной температуре.

С учётом термического расширения пористого тела формула (13) примет вид:

$$
q_{\text {крum }}=\frac{g\left[h_{\max }\left(\mathrm{T}_{i}\right) \frac{\sigma_{\varkappa}}{\sigma_{\varkappa}\left(\mathrm{T}_{i}\right)}-\rho_{\varkappa} L \sin \gamma\left(1+\beta^{\prime} \Delta \mathrm{T}\right)\right]}{\frac{L_{u} \mu_{\varkappa}}{r^{*} \rho_{ж} K^{\prime} r^{*} \xi b(1+\beta \Delta \mathrm{T})}\left(\frac{L_{u}}{2}+L_{a}\right)},
$$

где $\Delta \mathrm{T}=\mathrm{T}-\mathrm{T}_{i}$.

Приведенное уравнение хорошо согласуется с экспериментальными данными для воды вплоть до $q_{\text {крит }}=$ $=1300$ кВт $/ \mathrm{m}^{2}$, а для калия $q_{\text {крит }}=3150$ кВт/м². При более высоких величинах тепловых потоков лимитирующим является реактивное давление пара, выходящего из пор.

Высокие тепловые потоки при малых разностях температур в процессе кипения на пористых структурах объясняются тремя факторами:

- наличием внутри слоя границы раздела фазы, снижающей необходимый для парообразования перегрев;

- высоким коэффициентом конвективного теплообмена при ламинарном движении жидкости в капиллярном канале;

- развитой поверхностью капиллярной структуры.

При кипении жидкости на пористой поверхности имеется зона фазового перехода вблизи поверхности нагрева. Пар удаляется по большим порам, в то время как жидкость подсасывается к зоне фазового перехода с помощью капиллярных сил по малым порам.

\section{4. Теплообмен при кипении жидкости на раз- витой поверхности}

Кипение жидкости на развитой поверхности теплообмена (капиллярные канавки, винтовая нарезка, мелкие ребра, щели) может происходить как на затопленной, так и на незатопленной поверхности (жидкость находится в канавке). Последний случай широко ис- пользуется в тепловых трубах и паровых камерах, когда жидкость из конденсатора в испаритель подается по продольным канавкам или по пористым артериям и растекается под действием капиллярных сил по винтовой нарезке. Теплообмен в смоченных канавках испарителя происходит путем испарения жидкости с поверхности пленки либо путем кипения в канавках. Такие системы носят название тонкопленочных испарителей.

В ОНАПТ проводятся исследования процесса теплообмена при испарении и кипении жидкости в микроканальных испарителях [4 - 6]. Анализ экспериментальных данных показывает, что для достижения одного и того же значения теплового потока для поверхности с регулярной шероховатостью М-1 требуется перегрев на $2 \ldots 4{ }^{\circ} \mathrm{C}$ меньший по сравнению с гладкой профилированной пластинкой М-0, что естественно объясняется ростом количества центров парообразования. Визуальные наблюдения подтверждают, что процесс образования пузырьков на поверхности М-1 наступает значительно раньше (при $7 \mathrm{BT} / \mathrm{cm}^{2}$ и $5 \mathrm{BT} / \mathrm{cm}^{2}$ для воды и этанола, соответственно), по сравнению с поверхностью М-0 (12 Вт/см ${ }^{2}$ и $7 \mathrm{BT} / \mathrm{cm}^{2}$ для воды и этанола, соответственно). Гибридное сочетание поверхностей МС0.2 и МС-0.7 способствует улучшению теплопередающих характеристик за счёт увеличения капиллярности и обеспечения тонкопленочного испарения на поверхности сетки. Применение сетки позволяет удерживать жидкость, которая разбрызгивается вследствие разрыва пузырьков. При этом использование сеточной микроструктуры с равным или большим размером ячейки по сравнению с характерным размером канавки приводит к более интенсивному теплообмену, чем при использовании аналогичных структур, но с меньшим размером ячейки.

С целью исследования влияния формы и размеров поперечного сечения микроканалов на интенсивность процесса теплоотдачи была проведена серия опытов для 14 медных тонкопленочных испарителей с микроканалами прямоугольного, квадратного, треугольного, трапецеидального и круглого профиля. Определялось влияние на теплосъем геометрических параметров микроканала. Подвод тепловой мощности осуществлялся при граничных условиях третьего рода. В процессе экспериментов определялись суммарная тепловая мощность, снимаемая испарителем, и характер изменения температуры микроканала вдоль движения жидкости. Эксперименты проводились в диапазоне температур 283 - 363 К. Максимальные значения коэффициентов теплоотдачи (95 кВт/(м $\left.{ }^{2} \mathrm{~K}\right)$ при удельном тепловом потоке $130 \mathrm{BT} / \mathrm{cm}^{2}$ и27.5 кВт/(м² $)$ при $43 \mathrm{Bт} / \mathrm{cm}^{2}$ для воды и этанола, соответственно) обеспечивают каналы прямоугольного профиля П0,7*1,4*0,7, у которых глубина канала в 2 раза превосходит их ширину. Интересным представляется факт, что для прямоугольных каналов с одинаковой площадью поперечного сечения (П $0,3 * 0,6 * 0,3$ и П $0,3 * 0,3 * 0,6)$ коэффициент теплоотдачи примерно на 10-15\% выше, а передаваемый удельный тепловой на 10 Вт/см² больше для канала, у которого глубина в два раза превышает его ширину по сравнению с каналом, у которого ширина в 2 раза превосходит его глубину.

Выполнены расчеты оптимального угла при вершине треугольной канавки. Из условия равновесия сил на поверхности раздела фаз величина приращения гид- 
равлического сопротивления приравнивалась капиллярному напору:

$$
\xi \frac{\mu v}{2 D_{r}^{2}} d X=\frac{\sigma \cos \theta}{R_{\text {нап }}}
$$

В случае постоянства ширины микроканала скорость движения жидкости по нему в зависимости от угла при вершине равна

$$
v=-K \frac{\cos \alpha(1-\sin \alpha)}{1+\sin \alpha}
$$

При постоянной глубине

$$
q_{\text {max }}^{\prime}=\frac{\rho \mathrm{N}_{\varkappa} \cos \theta C(\alpha) K(\alpha)}{X_{\max }\left[X_{\max }-\left(X_{\max }+X_{0}\right)\left(1-\frac{1}{\left.\ln \frac{X_{0}+X_{\max }}{X_{0}}\right)}\right)\right.} ;
$$

где Хо - длина участка микроканала, занятого подводящей артерией, коэффициент $K(\alpha)$ учитывает влияние на теплообмен процесса кипения в микроканала. Для этанола экспериментально определено, что $K(\alpha)=0,05(1,8 \alpha)^{1,62}$.

Предельное значение теплового потока зависит от транспортной способности микроканала. Тенденция увеличения теплового потока определяется увеличением скорости протекания жидкости по микроканалу. Ограничением по теплообмену при больших плотностях потока является наступление режима пленочного кипения. Он характеризуется образованием устойчивой паровой прослойки у поверхности нагрева, которая препятствует поступлению туда жидкости.

\section{Выводы}

В ходе комплексных экспериментальнотеоретических исследований установлено, что микроканальные тонкопленочные испарители позволяют снимать тепловую мощность, близкую, а в некоторых случаях и большую той, которая обеспечивается при кипении жидкости в большом объеме.

Вместе с тем нужно указать, что на процесс кипения жидкости в микроканале оказывают воздействие многочисленные факторы: чистота обработки, смачивание, положение относительно поля гравитации, физические свойства жидкости и пара, геометрические размеры поверхности нагрева и т.д. Трудность количе- ственной оценки этого влияния препятствует подробному аналитическому описанию теплообмена при кипении движущейся жидкости в микроканале. В случае двухфазного потока, имеющего небольшую скорость перемещения, распределение пара и жидкости по сечению канала происходит неравномерно, что приводит к локальным перегревам, пульсациям давления по периметру канала.

Таким образом, можно утверждать, что процесс теплообмена при испарении и кипении жидкостей в капиллярно-пористых телах и на развитых поверхностях, покрытых сетью капиллярных каналов, обладает рядом особенностей по сравнению с кипением жидкости в большом объеме над гладкой поверхностью, а также в каналах и трубах с гладкими стенками. Пористая структура и капиллярные канавки интенсифицируют процесс теплообмена в широком диапазоне тепловых потоков и позволяют производить плавный переход от режима испарения к режиму кипения без звуковых эффектов и механических вибраций. Процесс наступления кризиса кипения сглаживается, при этом кривая кипения не имеет ярко выраженных максимумов. Покрытие пористыми структурами или микроканалами поверхности теплообмена с целью интенсификации процесса особенно эффективно для криогенных жидкостей (гелий, водород и т. д.), а также в низкотемпературных тепловых трубах и термосифонах.

\section{Литература}

1. Лыков А.В. Теория теплопроводности.- М.: Высшая школа, 1967.- 600 с.

2. Ягов В.В., Городов А.К., Лабунцов Д.А. Экспериментальное исследование теплообмена при кипении жидкостей при пониженных давлениях.- ИФЖ. 1970, T.18, №4.- C. 624-630.

3. Ferrell, J.K., \& Alleavitch, J. (1970). Vaporization heat transfer in capillary wick structures. Chem Eng Progr, Symp Ser, 66(102), 82-91.

4. Kosoy B.V., Peculiarities of heat transfer on microstructured surfaces / B.V. Kosoy, Y. Utaka // Proc. 50th National Heat Transfer Symposium of Japan, Sendai, Japan, May 29-31, 2013. - Vol. II+III, D211. - P. 342-343.

5. Experimental study of evaporation at microgroove surfaces [Text] / O. G. Burdo, B. V. Kosoy, G. F. Smirnov, M. I. Slobodeniuk // Heat Pipe Science and Technology, An International Journal - 2013. - Vol. 4. - P. 119-131.

6. Экспериментальное исследование критического теплового потока при кипении недогретого этанола в микроканалах [Текст] / М. И. Слободенюк, О. Г. Бурдо, А. А. Кондратенко, Б.В. Косой // Промышленная теплотехника. - 2013. - Т. 35 (7). - С.53-58.

\title{
Factors of boiling augmentation in two-phase thermal control systems
}

\author{
B. V. Kosoy
}

Odessa National Academy of Food Technologies, 112 Kanatna str., Odessa, 65039, Ukraine

e-mail: bkosoy@gmail.com

The present study is devoted to the experimental and theoretical solution of an important scientific and technical problem of the heat transfer augmentation in the microstructural elements of thermal control systems in order to increase their thermal efficiency, reliability, and reduce weight and dimensions. The main structural characteristics of capillary-porous bodies, such as effective porosity, curve of pores distribution against the radius, permeability, etc., are considered. The 
core mechanisms of mass transfer in a porous medium are determined. The analysis of the features of model representations for the processes of liquid boiling on porous and enhanced heat transfer surfaces is carried out, and physical factors are validated to ensure high heat fluxes at small temperature differences. The implemented complex of experimental and design studies of the two-phase heat transfer characteristics at boiling in microchannel thin-film evaporators made it possible to determine the specific influence of the surface structure on the intensity of the heat transfer process; the dependencies of their heat transfer capacity on the cross-sectional shape and the ratios between the depth and width of rectangular microchannels were established. A theoretical analysis of the characteristics of hydrodynamic and heat transfer processes occurring in microstructures was carried out, demonstrating the possibilities of boiling heat transfer enhancement by optimizing the thermal characteristics of the microstructure and using hybrid microstructures of various porosities. It has been established that heat transfer at evaporation and boiling of liquids in capillary-porous media and on ehanced surfaces covered with a network of capillary channels has a number of features compared to boiling a liquid in a large volume over a smooth surface, as well as in channels and pipes with flat walls. The porous structure and capillary grooves intensify the heat transfer in a wide range of heat fluxes and allow a smooth transition from evaporation to boiling. The process of the onset of the boiling crisis is smoothed, while the boiling curve does not have pronounced maxima. Coating with porous structures or microchannels of the heat transfer surface to intensify the process is especially effective for cryogenic liquids (helium, hydrogen, etc.), as well as in low-temperature heat pipes and thermosyphons.

Keywords: Augmentation; Boiling; Thermal control; Microchannels; Porous media

\section{References}

1. Lykov, A. V. (1967) Teoriia teploprovodnosti. Moskow: Vysshaia shkola, $600 \mathrm{p}$.

2. Ягов В.В., Городов А.К., Лабунцов Д.А. Экспериментальное исследование теплообмена при кипении жидкостей при пониженных давлениях.- ИФЖ. 1970, 18(4), 624-630. (in Russian)

3. Ferrell, J.K., \& Alleavitch, J. (1970). Vaporization heat transfer in capillary wick structures. Chem Eng Progr, Symp Ser, 66(102), 82-91.

4. Kosoy, B. V., Utaka, Y. Peculiarities of heat transfer on micro-structured surfaces. Proc. 50th National Heat Transfer Symposium of Japan, Sendai, Jappan
May 29-31, 2013. Vol. II+III, D211, pp. 342-343.

5. Burdo, O. G., Kosoy, B. V., Smirnov, G. F., Slobodeniuk, M. I. (2013) Experimental study of evaporation at microgroove surfaces. Heat Pipe Science and Technology, An International Journal, 4 (1-2), 119-131. doi: https://doi.org/10.1615/heatpipescietech.2013007827

6. Slobodeniuk, M. I., Burdo, O. G., Kondratenko, A. A., Kosoy, B. V. (2013) Eksperimentalnoie issledvanie kriticheskogo teplovogovteplovogo potoka pri kipenii nedogretogo etanola v mikrokanale. Industrial Heat Engineering, 35 (7), 53-58. 